\title{
Numerical simulation of an acoustic field generated by a phased arc array in a fluid-filled cased borehole
}

\author{
Che Xiaohua ${ }^{1,2 *}$, Qiao Wenxiao ${ }^{1,2}$, Wang Ruijia ${ }^{1,2}$ and Zhao Yuhong ${ }^{3}$ \\ ${ }^{1}$ State Key Laboratory of Petroleum Resources and Prospecting, China University of Petroleum, Beijing 102249, China \\ ${ }^{2}$ Key Laboratory of Earth Prospecting and Information Technology, China University of Petroleum, Beijing 102249, China \\ ${ }^{3}$ China National Oil \& Gas Exploration \& Development Company, Beijing 100044, China
}

(C) China University of Petroleum (Beijing) and Springer-Verlag Berlin Heidelberg 2014

\begin{abstract}
Transducers that are widely applied in cement bond evaluation tools, such as cement bond logs and variable density logs, cannot radiate acoustic energy directionally because of the characteristics of monopole sources. A phased arc array transmitter, which is a novel transducer that differs from monopole and dipole transducers, is presented in this study. To simulate the acoustic field generated by a phased arc array in a fluid-filled cased borehole with different channelings, a 3D finite-difference time-domain method is adopted. The acoustic field generated by a traditional monopole source is also simulated and compared with the field generated by the phased arc array transmitter. Numerical simulation results show that the phased arc array radiates energy directionally in a narrow angular range in the borehole, thereby compressing the acoustic energy into a narrow range in the casing pipe, the cement, and the formation. We present the analyses of first-arrival waveforms and the amplitudes of casing waves at different azimuthal angles for the two different sources. The results indicate that employing a directional source facilitates azimuthal identification and analysis of possible channeling behind the casing pipe.
\end{abstract}

Key words: Phased arc array, azimuthal angle, first-arrival amplitude, acoustic field, cased borehole

\section{Introduction}

The acoustic properties of transducers determine the functions and detection characteristics of acoustic well logging tools, and essentially produce the new generation of downhole tools (Qiao et al, 2011). Monopole transducers were used in early acoustic well logging tools and are still being used at present. The significant radiation characteristic of monopole transducers used in well logging tools is their mono-directional horizontal directivity. Later on, multipole sources have been studied and applied to geophysical measurement practices (Chen and Tang, 2012; Kurkjian and Chang, 1986; Kessler and Varsamis, 2001; Markova et al, 2010; Tang and Cheng, 2004; Tang, 2004; Tang et al, 2013a; Wang et al, 2011; Wang et al, 2013). Combining a monopole source with quadrupole and dipole sources in acoustic well logging tools expands the functions and application scope of this logging technology. Unlike a monopole source, the directivity curve of a dipole source is $\infty$ shaped, thus extending acoustic tool functions. Dipole sources are used in the new acoustic logging technology of remote acoustic reflection imaging (Tang and Wei, 2012a; 2012b; Tang et al,

*Corresponding author. email: aclab@cup.edu.cn

Received August 21, 2013 2013b). However, azimuthal detection resolution for these tools is limited by the radiation directivity of dipole sources. As complex reservoirs are explored and developed, the need for azimuthal acoustic logging becomes urgent ( $\mathrm{Li}$ and $\mathrm{Chu}$, 1997). For cased boreholes, detecting azimuthal direction for channeling is crucial, but still faces difficulties.

Traditional acoustic monopole sources are still extensively used in traditional cement bond evaluation tools such as cement bond $\log (\mathrm{CBL})$ and variable density $\log$ (VDL). However, acoustic monopole sources cannot be employed to evaluate the azimuthal direction of channeling, partial channeling, and partial cement bonds. A segmented bond tool (SBT) can identify channeling, as well as evaluate channeling size and its azimuth. However, if SBT is used alone downhole, only the bond condition of the first interface (i.e., the interface between the cased pipe and the cement) can be assessed. To obtain the information on the second interface (i.e., the interface between the cement and the formation), SBT generally combined with CBL and VDL. Schlumberger developed a new-generation tool called Isolation Scanner, which evaluates strip channeling when cements with different densities are bonded (Bellabarba et al, 2008). This tool is a new well logging technology that combines ultrasonic reflection, ultrasonic refraction, CBL, and VDL. Downhole well logging tools with dipole sources are highly effective 
in evaluating formations through casing (Che et al, 2007; Tang and Cheng, 2004) but not in evaluating cement bonds. Linear phased array transmitters have been mainly used in open boreholes (Che and Qiao, 2004; Qiao et al, 2002; Qiao et al, 2003; Zhang et al, 2004). These transmitters have also been studied for enhancing the amplitude of formation waves in cased boreholes (Chen and Qiao, 2007; Chen and $\mathrm{Xu}, 2008)$. Similar to a dipole transducer, a linear phased array transmitter is inappropriate for evaluating azimuthal cement bonds. Using a new source with directional radiation properties in downhole tools (Qiao et al, 2006) provides a new well logging technology with azimuthal directional resolution that can be used in cased holes. Qiao et al studied the radiation directivity of a phased arc array transmitter (Qiao et al, 2008; Wu et al, 2013) and the acoustic field generated in a fluid-filled open hole (Che and Qiao, 2009) but not in a cased hole. These researchers showed that the acoustic waves generated by an array transmitter in a fluid-filled borehole also include refracted $\mathrm{P}$ and $\mathrm{S}$ waves of the formation, which is the theoretical foundation of phased arc array acoustic logging.

The finite-difference time-domain (FDTD) method is extensively used to simulate acoustic fields in acoustic well logging (He et al, 2006; He et al, 2010; Lin et al, 2006; Liu et al, 1996; Liu and Sinha, 2000; Mittet and Renlie, 1996; Randall et al, 1991; Stephen et al, 1985; Yang et al, 2009). In the current work, we adopt a 3D FDTD method to simulate the acoustic field generated by a phased arc array transmitter in a fluid-filled cased borehole with channeling. We then compare the generated acoustic field with that generated by a traditional monopole source.

\section{Finite-difference method}

The 3D FDTD method is useful in cases when wave equations do not have an analytical solution. In the current study, 3D finite-difference algorithms are described in Cartesian coordinates. The main equations employed are obtained from previous studies (Che and Qiao, 2009; Lin et al, 2006; Liu et al, 1996).

To simulate models numerically with finite media, we reduce reflections from artificial boundaries. Berenger introduced the perfectly matched layer (PML) as an effective absorbing boundary condition for electromagnetic waves (Berenger, 1994). The PML for elastic waves is a perfectly matched layer to absorb outgoing waves without reflections on the artificial boundaries of the computational region (Chew and Liu, 1996; Liu, 1999; He and Hu, 2009; He et al, 2010). In the present study, the splitting PML is employed as the absorbing boundary condition based on previous studies (Chew and Liu, 1996; Collino and Tsogka, 2001). Stiffness coefficients and densities are averaged by adjacent components (Cheng et al, 1995; He et al, 2010). In Cartesian coordinates, the stable condition used in this study satisfies the following equation:

$$
\left\{\begin{array}{l}
\frac{\Delta t v_{\max }}{\Delta x} \leq\left(\sqrt{3} \sum_{m=0}^{N-1}\left|a_{m}\right|\right)^{-1} \\
\sqrt{(\Delta x)^{2}+(\Delta y)^{2}+(\Delta z)^{2}}<\frac{v_{\min }}{2 f_{\max }}
\end{array}\right.
$$

where $v_{\max }$ and $v_{\min }$ are the fastest and the slowest velocity in the model, respectively; $a_{m}$ is the coefficient of the finite difference in the calculation; $N$ is a variable, and the value of $N$ is half of the order of FDTD method in space; $\Delta x$, $\Delta y$, and $\Delta z$ are the space steps in the $x, y$, and $z$ directions, respectively; $f_{\max }$ is the maximum frequency of the source; and $\Delta t$ is the time step.

Calculations of 3D models are limited by computer memory size, CPU speed and disk space. Parallel computing provides an efficient means to calculate 3D problems through the finite-difference method. All models are executed on a high performance cluster computer system. The programming technologies of Open Multi-Processing (OpenMP) and Message Passing Interface (MPI) are combined to simplify the complexity of the parallel algorithm and enhance program execution efficiency.

\section{The acoustic field generated by a phased arc array in a cased borehole}

\subsection{The calculation model}

The horizontal section of an eight-element phased arc array transmitter is shown in Fig. 1, where A to $\mathrm{H}$ represent eight piezoelectric elements that are evenly positioned around a circle. The transmitter can be approximated as a traditional monopole source if elements A to $\mathrm{H}$ are simultaneously fired. By contrast, the transmitter works as a phased arc array if only several elements are excited. For example, if three adjacent elements are selected as one sub-array, then the transmitter will function as a phased arc array source. Thus, the eight-element phased arc array transmitter can be considered as eight three-element sub-arrays. We provide an example to demonstrate how the three-element sub-array with elements $\mathrm{B}, \mathrm{A}$ and $\mathrm{H}$ is fired. Elements $\mathrm{B}$ and $\mathrm{H}$ are simultaneously excited at time zero, whereas element $\mathrm{A}$ is excited at time $\tau . \tau$ is determined by the propagation time of the acoustic waves from element $\mathrm{B}$ or $\mathrm{H}$ to element $\mathrm{A}$. The wavefronts generated by elements $\mathrm{B}$ and $\mathrm{H}$ simultaneously arrive at element $\mathrm{A}$ at moment $\tau$. The main energy of the subarray radiates and propagates near elements $\mathrm{B}, \mathrm{A}$, and $\mathrm{H}$. A similar firing scheme is executed for other three-element sub-arrays, and the main energy radiates toward different directions. The acoustic signals of the sub-arrays can be driven one after another by an electronic control system; thus, acoustic energy is radiated and azimuthal scanning is enabled. Azimuthal scanning by using the phased arc array radiates acoustic energy onto the casing at an angle interval of $45^{\circ}$. The waveform of the acoustic source and its corresponding amplitude spectrum are shown in Fig. 2. The source signal is a Ricker wavelet, and the central frequency of the acoustic pulse source is $16 \mathrm{kHz}$.

The dimensions of the cylindrical model well are as follows: height $\times$ diameter, $3.8 \mathrm{~m} \times 1.0 \mathrm{~m}$; borehole radius, $10 \mathrm{~cm}$; casing thickness, $1.0 \mathrm{~cm}$; annular cement thickness, $2.0 \mathrm{~cm}$. The compressional wave and shear wave velocities of the casing are $5,900 \mathrm{~m} / \mathrm{s}$ and $3,230 \mathrm{~m} / \mathrm{s}$, respectively; the casing density is $7,800 \mathrm{~kg} / \mathrm{m}^{3}$. The compressional wave and shear wave velocities of cement are $3,500 \mathrm{~m} / \mathrm{s}$ and 2,000 


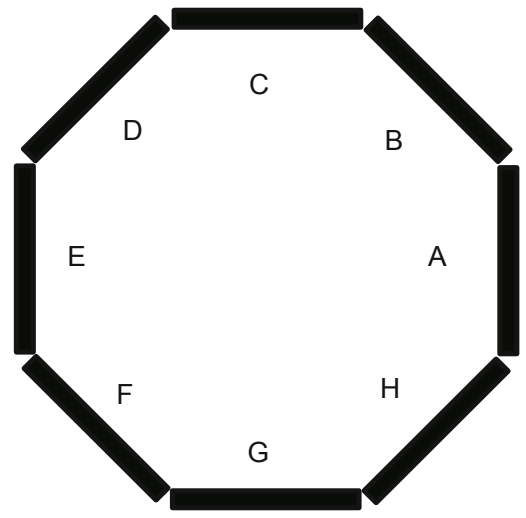

Fig. 1 Horizontal section of the acoustic source

$\mathrm{m} / \mathrm{s}$, respectively, with a cement density of $1,900 \mathrm{~kg} / \mathrm{m}^{3}$. The compressional wave and shear wave velocities of the formation are $4,000 \mathrm{~m} / \mathrm{s}$ and $2,500 \mathrm{~m} / \mathrm{s}$, respectively. The formation density is $2,200 \mathrm{~kg} / \mathrm{m}^{3}$, the fluid velocity is 1,500 $\mathrm{m} / \mathrm{s}$, and the fluid density is $1,000 \mathrm{~kg} / \mathrm{m}^{3}$.

The sketch map of the transmitter and receiver system in the model well is shown in Fig. 3(a). The phased arc array transmitter $(\mathrm{T})$ and the eight-element receiver $(\mathrm{R})$ are in the fluid-filled borehole, the eight receivers are at the same height in the $z$ axis but in different circumferential positions. Eight waveforms can be obtained by the eight-element receiver when the transmitter works once. Different waveforms can be used to analyze the transmitter radiation characteristics in the circumferential direction. Channeling is an important problem in cementing a casing to the formation as voids and channels can remain unfilled by cement. Assuming that a strip

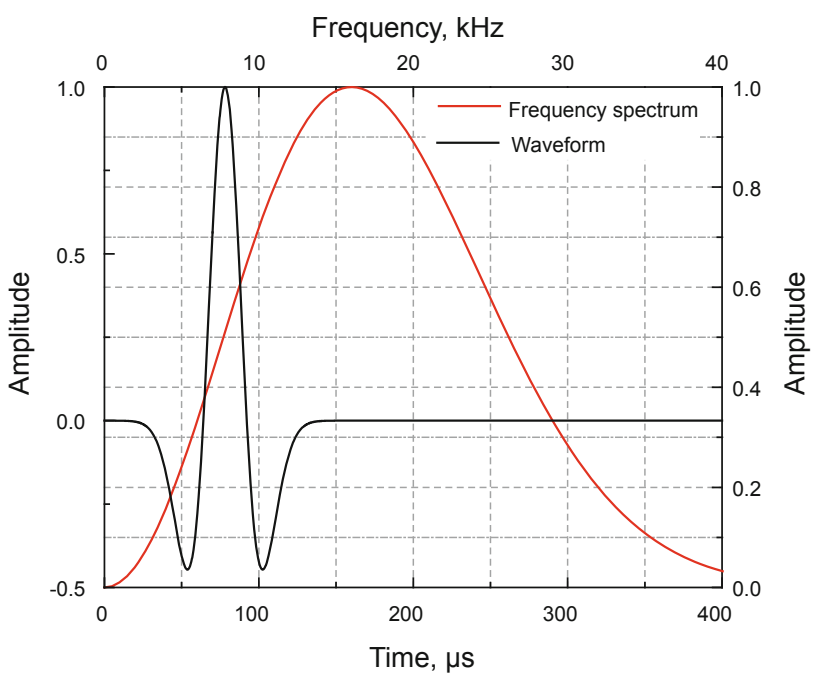

Fig. 2 Waveform of the acoustic source and its corresponding amplitude spectrum

channeling in the cement is located between the transmitter and the receivers, the azimuthal angle width of the channeling is $\beta$, and its vertical axial height is $0.5 \mathrm{~m}$. The horizon-slice map of the phased arc array transmitter and the model well with cement strip channeling is shown in Fig. 3(b). The angle between the main radiation beam direction of the threeelement sub-array and the cement strip channeling center is $\alpha . \alpha$ can be theoretically assigned to any value. However, considering practical significance and the realization of the electrical circuit, the discrete values of $\alpha$ are set to $0^{\circ}, 45^{\circ}$, $90^{\circ}, 135^{\circ}, 180^{\circ}, 225^{\circ}, 270^{\circ}$, and $315^{\circ}$ in this study.

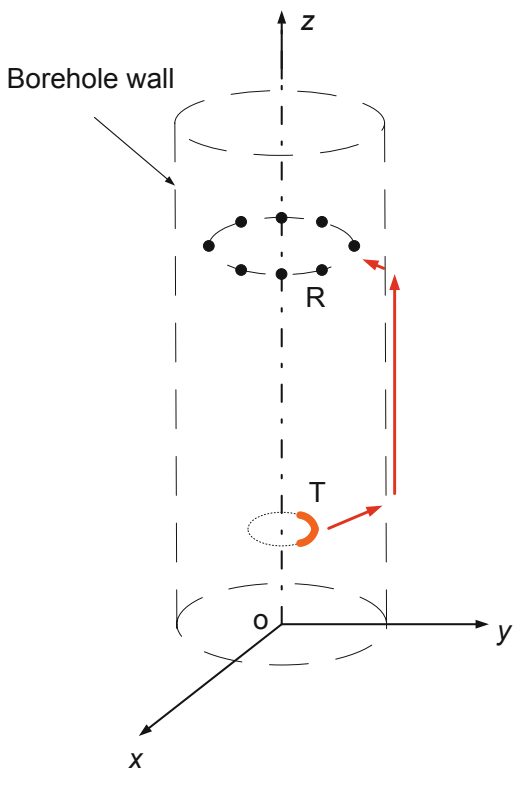

(a)

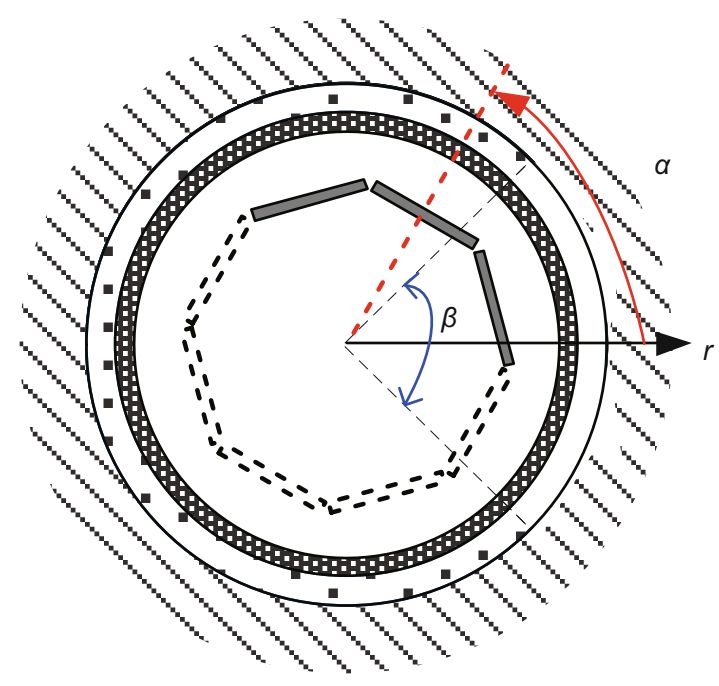

(b)

Fig. 3 Transmitter and receiver system of the model well

(a) Sketch map of the transmitter and receiver in the borehole and (b) Horizontal-slice map of the phased arc array transmitter and the model well with cement strip channeling 


\subsection{Numerical simulation results}

For a model with $\beta=90^{\circ}$, the monopole source and the phased arc array individually radiate acoustic energy at $\alpha$ $=0^{\circ}$. The eight first arrivals received by the eight-element receiver are shown in Fig. 4, wherein the spacing is $3 \mathrm{ft}$. The main lobe direction of the radiation from the phased arc array coincides with the circumferential center of the cement channeling when $\alpha=0^{\circ}$.

The relationship curve between the amplitudes of the first arrivals and the azimuthal directions of the receiver is based on all first-arrival amplitudes of the received waveforms (Fig. 5). The curve labeled "TM-RA" is for the monopole source,

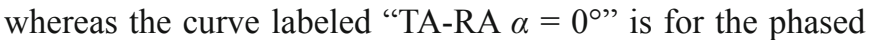
arc array. As shown in Figs. 4 and 5, the first arrivals received by the eight-element receiver are obviously associated with the azimuthal directions of the receiver. The received acoustic lobe exhibits a strong directional property at $\beta=90^{\circ}$. The

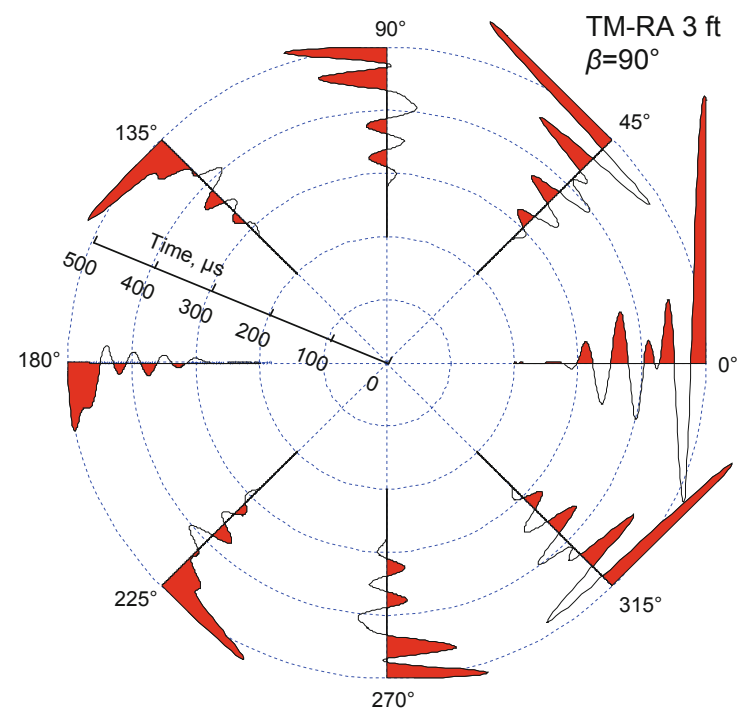

(a)

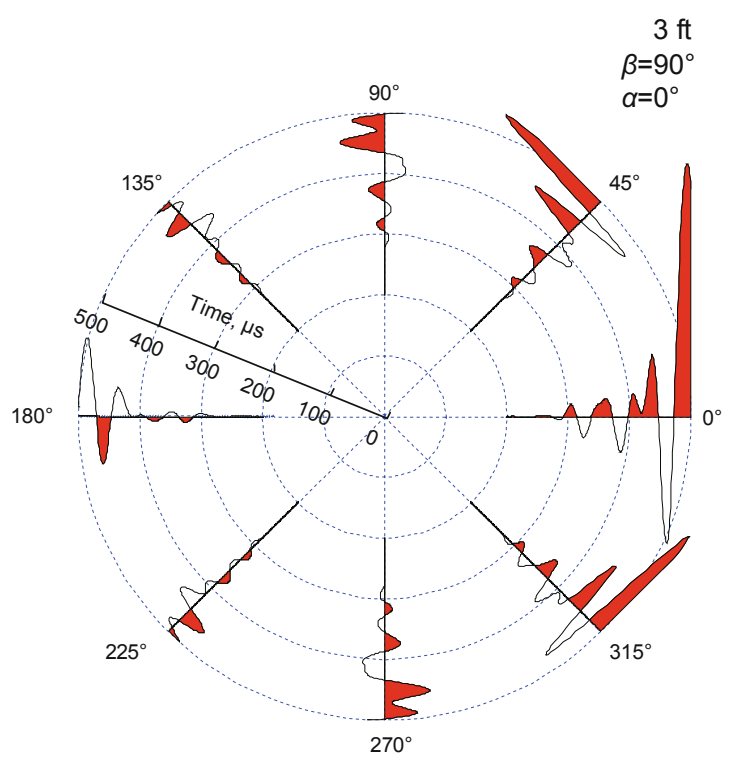

(b)

Fig. 4 Eight first arrivals received by the eight-element receiver (a) the monopole source and (b) the phased arc array radiation at $\alpha=0^{\circ}$ differences in amplitudes of the first arrivals received by different elements of the receiver when the transmitter is used as a phased arc array are all larger than those received when the source is a traditional monopole. More models for $\beta=0^{\circ}$, $27^{\circ}$, and $45^{\circ}$ are calculated, but are not presented in this paper because similar conclusions are reached. When the phased arc array is azimuthally scanned, $\alpha$ can be $0^{\circ}, 45^{\circ}, 90^{\circ}, 135^{\circ}$, $180^{\circ}, 225^{\circ}, 270^{\circ}$, and $315^{\circ}$. The waveforms received by the element that aligns with the azimuthal center of the cement channeling, when azimuthally scanned acoustic energy of the phased arc array is at $\beta=90^{\circ}$, are shown in Fig. 6 . The amplitude of the received signal is highest when the radiated acoustic energy of the phased arc array is at $\alpha=0^{\circ}$ (aligns with the channeling direction; Fig. 6).

The curves that represent the relationship between the first-arrival amplitudes and the eight azimuthal directions of the receiver at $\beta=0^{\circ}, 27^{\circ}, 45^{\circ}$, and $90^{\circ}$ when the phased arc array radiation is at $\alpha=0^{\circ}$ are shown in Figs. 7(a) and 7(b). The curves in Fig. 7(a) are not normalized, whereas those in Fig. 7(b) are normalized. The figures show visible differences between the eight first arrivals received by the different elements of the receiver and the azimuthal directions of the receiver. The received acoustic beam has a clearly observable directional property. In addition, the angle width of the main lobe becomes narrow when the azimuthal angle of the channeling increases, thus indicating that a large channeling is more easily detected than a small channeling. When the direction of the main radiation beam of the three-element subarray is facing the cement strip channeling center ( $\alpha$ is equal to $0^{\circ}$ ), the difference between the first-arrival amplitudes on the eight azimuthal directions is the highest. Otherwise $(\alpha$ is not equal to $0^{\circ}$ ), the difference between the first-arrival amplitudes on the eight azimuthal directions decreases. The aforementioned relative difference of first arrivals is used in applications to detect the azimuth of the cement strip channeling.

The curves of the relationship between the first-arrival amplitudes and the eight azimuthal directions of the receiver

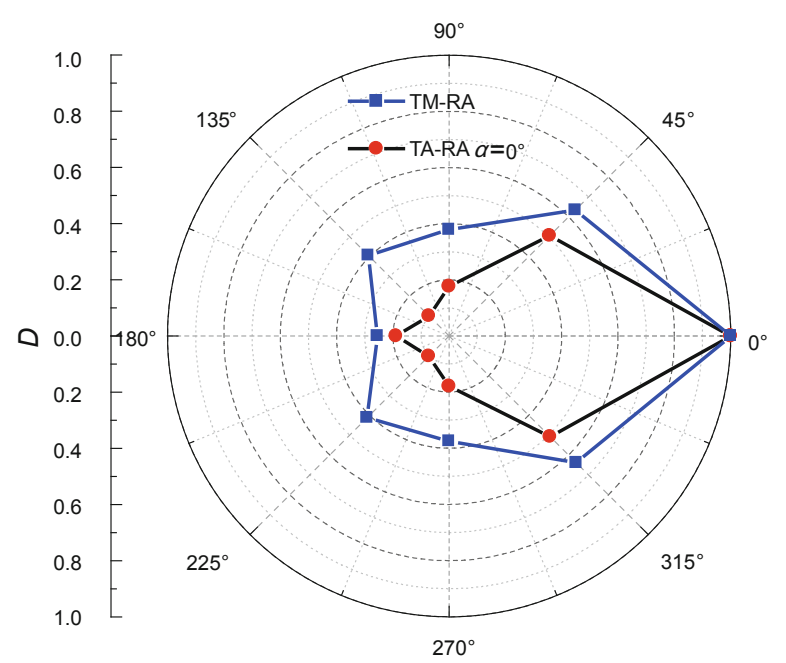

Fig. 5 Relationship curves of the first-arrival amplitudes and the azimuthal directions of the receiver (based on the first-arrival amplitudes of the received waveforms in Fig. 4). $D$ is a dimensionless variable 


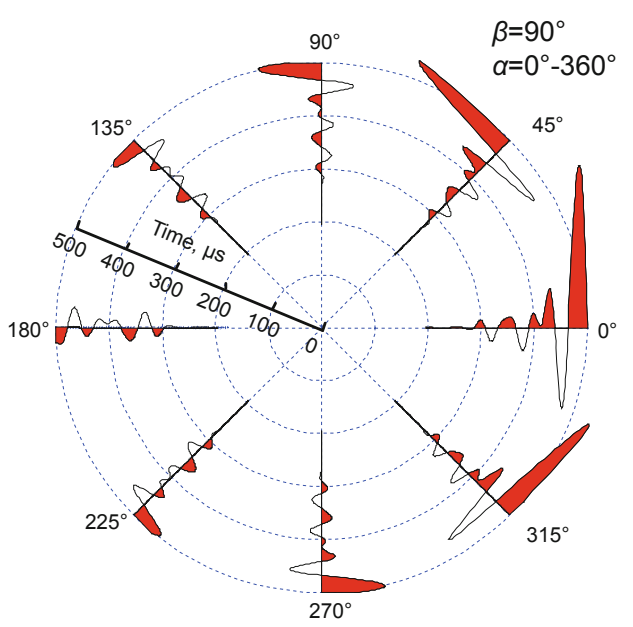

Fig. 6 Received waveforms when the azimuthally scanned acoustic energy of the phased arc array is at $\beta=90^{\circ}$, the receiver aligns with the azimuthal center of the cement channeling

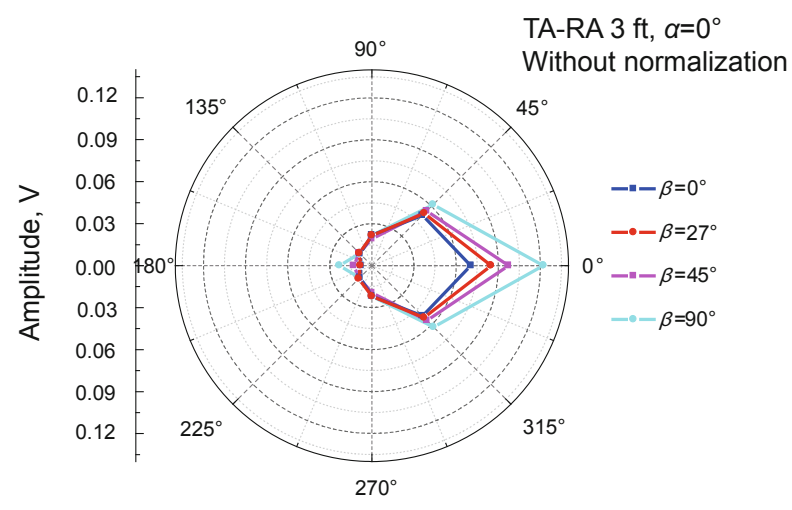

(a)

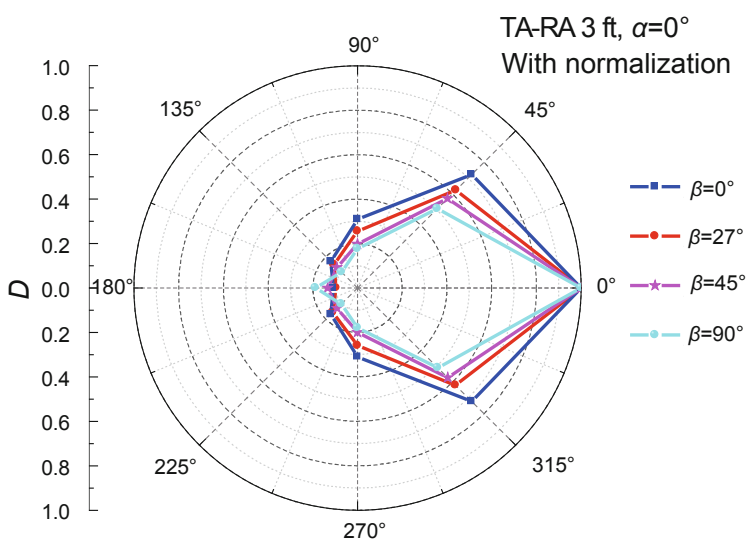

(b)

Fig. 7 Relationship curves of the first-arrival amplitudes and the eight azimuthal directions of the receiver at $\beta=0^{\circ}, 27^{\circ}, 45^{\circ}$, and $90^{\circ}$ when the phased arc array radiation is at $\alpha=0^{\circ}$. (a) without normalization and (b) with normalization. $D$ is a dimensionless variable

under monopole source radiation are shown in Fig. 8. Obvious differences are observed between the eight first arrivals received by the different elements of the receiver and the azimuthal directions of the receiver. The received acoustic beam exhibits an apparent directional property, and the angle width of the main lobe becomes narrow when the azimuthal angle of the channeling increases. However, the angle width of the received acoustic main lobe is considerably wider than that of the phased arc array radiation. This result indicates that the azimuthal detection resolution of the phased arc array radiation is higher than that of the monopole source radiation.

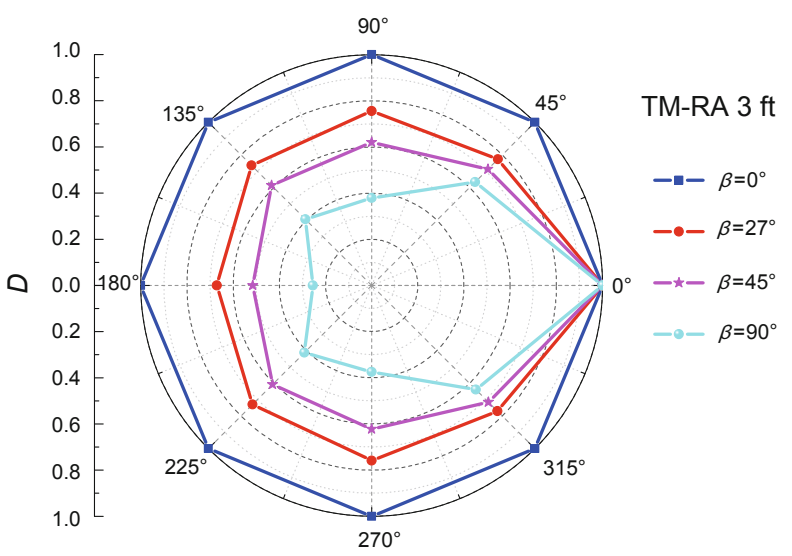

Fig. 8 Relationship curves of the first-arrival amplitudes and the eight azimuthal directions of the receiver at $\beta=0^{\circ}, 27^{\circ}, 45^{\circ}$, and $90^{\circ}$ under a monopole source radiation. $D$ is a dimensionless variable

\section{Conclusions}

We have numerically simulated the acoustic field generated by a phased arc array and a traditional monopole source in a fluid-filled cased borehole by using a 3D FDTD method in Cartesian coordinates. All the 3D models are executed on a high performance cluster computer system. The programming technologies OpenMP and MPI are combined to simplify the complexity of the parallel algorithm and enhance program execution efficiency. The numerical simulation results show that the acoustic energy generated by the phased arc array is radiated primarily into a narrow azimuthal range. Different cement bond model wells exhibit obvious variations among the eight first arrivals received by different elements of the receiver. Changes in the first arrivals along the eight directions of the eight-element receiver are apparent. The received acoustic beam exhibits a directional property, and the angle width of the main lobe becomes narrow as the azimuthal angle of the channeling increases. The angle width of the received acoustic main lobe is considerably wider than that of the phased arc array radiation, whereas the relative differences among the eight first arrivals are smaller. Therefore, the azimuthal detection resolution of the phased arc array radiation is higher than that of the monopole source radiation. The azimuthal direction of a bad cement bond can be accurately located by using an acoustic scanning radiation technology based on a phased arc array. Thus, phased arc array technology with directional radiation and reception functions is obviously one of the best options for evaluating cement channeling strips.

\section{Acknowledgements}

This work is supported by the National Natural Science 
Foundation of China (Grant Nos. 11204380, 11374371, 11134011 and 61102102), National Science and Technology Major Project (Grant No. 2011ZX05020-009) and PetroChina Innovation Foundation (2013D-5006-0304). The authors are also grateful to the editors and reviewers for their valuable comments.

\section{References}

Bellabarba M, Bulte-Loyer H, Froelich B, et al. Ensuring zonal isolation beyond the life of the well. Oilfield Review. 2008. 20: 18-31

Berenger J P. A perfectly matched layer for the absorption of electromagnetic waves. Journal of Computational Physics. 1994. 114(2): $185-200$

Che X H and Qiao W X. Acoustic field in formation generated by linear phased array transmitters in fluid-filled boreholes. Chinese Journal of Geophysics. 2004. 47(4): 830-836 (in Chinese)

Che X H and Qiao W X. Numerical simulation of an acoustic field generated by a phased arc array in a fluid-filled borehole. Petroleum Science. 2009. 6: 225-229

Che X L, Chen X X, Wang R J, et al. Study on the application of the dipole source in the cased-hole acoustic logging. Well Logging Technology. 2007. 31(3): 245-248 (in Chinese)

Chen X L and Qiao W X. Acoustic field in cased well excited by the linear phased-array transmitter. Acta Acustica. 2007. 32(4): 328-332 (in Chinese)

Chen X L and Tang X M. Numerical study on the characteristics of acoustic logging response in the fluid-filled borehole embedded in crack-porous medium. Chinese Journal of Geophysics. 2012. 55(6): 2129-2140 (in Chinese)

Chen X L and Xu X. Application of linear phased-array technique to estimation of acoustic properties of formation in acoustic logging of cased hole. Acta Petrolei Sinica. 2008. 29(5): 777-781 (in Chinese)

Cheng N, Cheng C H and Toksöz M N. Borehole wave propagation in three dimensions. J. Acoust. Soc. Am. 1995. 97: 3483-3493

Chew W C and Liu Q H. Perfectly matched layers for elastodynamics: A new absorbing boundary conditions. J. Computat. Acoustics. 1996. 4(4): 341-359

Collino F and Tsogka C. Application of the perfectly matched absorbing layer model to the linear elastodynamic problem in anisotropic heterogeneous media. Geophysics. 2001. 66(1): 294-307

He F, Tao G and Wang X L. Finite difference modeling of the acoustic field by sidewall logging devices. Chinese Journal of Geophysics. 2006. 49(3): 923-928 (in Chinese)

$\mathrm{He} \mathrm{X}$ and $\mathrm{Hu} \mathrm{H}$. Borehole flexural modes in transversely isotropic formations: the low-frequency asymptotic velocity. Geophysics. 2009. 74(4): E149-E158

$\mathrm{He} \mathrm{X}, \mathrm{Hu} \mathrm{H}$ and Guan W. Fast and slow flexural waves in a deviated borehole in homogeneous and layered anisotropic formations. Geophys. J. Int. 2010. 181(1): 417-426

Kessler C and Varsamis G L. A new generation crossed dipole logging tool: design and case histories. SPE Annual Technical Conference and Exhibition, 30 September - 3 October 2001, New Orleans, Louisiana (SPE 71740)

Kurkjian A L and Chang S K. Acoustic multipole sources in fluid-filled boreholes. Geophysics. 1986. 51(1): 148-163

Li Z and Chu Z. Present situation and tendency of research on well logging in China. Chinese Journal of Geophysics. 1997. 40(Supp. 1): 333-343 (in Chinese)

Lin W J, Wang X M and Zhang H L. Acoustic wave propagation in a borehole penetrating an inclined layered formation. Chinese Journal of Geophysics. 2006. 49(1): 284-294 (in Chinese)

Liu Q H. Perfectly matched layers for elastic waves in cylindrical and spherical coordinates. J. Acoust. Soc. Am. 1999. 105(4): 2075-2084

Liu Q H and Sinha B K. Multipole acoustic waveforms in fluid-filled boreholes in biaxially stressed formations: A finite-difference method. Geophysics. 2000. 65(1): 190-201

Liu Q H, Schoen E, Daube F, et al. A three-dimensional finite difference simulation of sonic logging. J. Acoust. Soc. Am. 1996. 100(1): 72-79

Markova I, Sadovnychiy S and Markov M. Acoustical multipole source in a hollow fluid-filled cylinder. International Journal of Engineering Science. 2010. 48(5): 507-517

Mittet R and Renlie L. High-order finite-difference modeling of multipole logging in formations with anisotropic attenuation and elasticity. Geophysics. 1996. 61(1): 21-33

Qiao W X, Che X H, Ju X D, et al. Acoustic logging phased arc array and its radiation directivity. Chinese Journal of Geophysics. 2008. 51(3): 939-946 (in Chinese)

Qiao W X, Chen X L, Du G S, et al. Laboratory simulation on acoustic well-logging with phased array transmitter. Acta Acustica. 2003. 22(4): 329-338

Qiao W X, Du G and Chen X L. Feasibility of application of linear phased array transmitters to acoustic well-logging. Chinese Journal of Geophysics. 2002. 45(5): 755-763 (in Chinese)

Qiao W X, Ju X D, Che X H, et al. Transducers and acoustic well logging technology. Physics. 2011. 40(2): 99-106 (in Chinese)

Qiao W X, Ju X D, Chen X L, et al. Downhole acoustic arc array with controlled azimuthal directivity. China Patent ZL. 2006. 03137596.0 (in Chinese)

Randall C J, Scheibner D J and Wu P T. Multipole borehole acoustic waveforms: synthetic logs with beds and borehole washouts. Geophysics. 1991. 56(11): 1757-1769

Stephen R A, Cardo-Casas F and Cheng C H. Finite-difference synthetic acoustic logs. Geophysics. 1985. 50(10): 1588-1609

Tang X M. Imaging near-borehole structure using directional acousticwave measurement. Geophysics. 2004. 69(6): 1378-1386

Tang X M and Cheng A. Quantitative Borehole Acoustic Methods. Netherlands: Elsevier Publishing Co. 2004

Tang X M and Wei Z. Significant progress of acoustic logging technology: remote acoustic reflection imaging of a dipole acoustic system. Applied Acoustics. 2012a. 31(1): 10-17 (in Chinese)

Tang X M and Wei Z. Single-well acoustic reflection imaging using farfield radiation characteristics of a borehole dipole source. Chinese Journal of Geophysics. 2012b. 55(8): 2798-2807 (in Chinese)

Tang X M, Cao J J and Wei Z T. Radiation and reception of elastic waves from a dipole source in open and cased boreholes. Journal of China University of Petroleum. 2013a. 37(5): 57-64 (in Chinese)

Tang X M, Wei Z T, Su Y D, et al. A new acoustic remote sensing method utilizing borehole low-frequency dipole shear wave. Chinese Journal of Geophysics. 2013b. 56(10): 3572-3580 (in Chinese)

Wang R J, Qiao W X, Ju X D, et al. Experimental study of the acoustic field in the borehole surrounded by HTI formations excited by dipole sources with different orientations. Chinese Journal of Geophysics. 2013. 56(2): 707-717 (in Chinese)

Wang X M, Zhang H L, He X, et al. Physical problems in acoustic logging. Physics. 2011. 40(2): 79-87 (in Chinese)

Wu J P, Qiao W X, Che X H, et al. Experimental study on the radiation characteristics of downhole acoustic phased combined arc array transmitter. Geophysics. 2013. 78(1): D1-D9

Yang Y, Che X H, Zhang F, et al. Sound isolation numerical simulation on isolator of logging while drilling sonic tool using finite difference time-domain method. Journal of China University of Petroleum. 2009. 33(3): 66-70 (in Chinese)

Zhang H L, Wang X M and Zhang B X. Acoustic Fields and Waves in Boreholes. Beijing: Science Press. 2004 (in Chinese)

(Edited by Hao Jie) 